\title{
Effects of relevant and irrelevant color singletons on inhibition of return and attentional capture
}

\author{
Ulrich Ansorge • Heinz-Werner Priess • Dirk Kerzel
}

Published online: 12 September 2013

(C) Psychonomic Society, Inc. 2013

\begin{abstract}
We tested whether color singletons lead to saccadic and manual inhibition of return (IOR; i.e., slower responses at cued locations) and whether IOR depended on the relevance of the color singletons. The target display was preceded by a nonpredictive cue display. In three experiments, half of the cues were response-relevant, because participants had to perform a discrimination task at the cued location. With the exception of Experiment 2, none of the cue colors matched the target color. We observed saccadic IOR after color singletons, which was greater for slow than for fast responses. Furthermore, when the relevant cue color matched the target color, we observed attentional capture (i.e., faster responses at cued locations) with rapid responses, but IOR with slower responses, which provides evidence for attentional deallocation. When the cue display was completely response-irrelevant in two additional experiments, we did not find evidence for IOR. Instead, we found attentional capture when the cue color matched the target color. Also, attentional capture was greater for rapid responses and with short cue-target intervals. Thus, IOR emerges when cues are relevant and do not match the target color, whereas attentional capture emerges with relevant and irrelevant cues that match the target color.
\end{abstract}

U. Ansorge $\cdot$ H.-W. Priess

Fakultät für Psychologie, Universität Wien, Vienna, Austria

\section{U. Ansorge}

Institut für Kognitionswissenschaften, Universität Osnabrück, Osnabrück, Germany

\section{Kerzel}

Faculté de Psychologie et des Science de l'Éducation, Université de Genève, Geneva, Switzerland

U. Ansorge $(\bowtie)$

Fakultät für Psychologie, Universität Wien, Liebiggasse 5, 1010 Vienna, Austria

e-mail: ulrich.ansorge@univie.ac.at
Keywords Attention $\cdot$ Saccades $\cdot$ Cueing $\cdot$ Inhibition of return

\section{Introduction}

Visual foraging behavior benefits from a track record of recently inspected locations. One mechanism serving this aim is inhibition of return (IOR; Wang \& Klein, 2010). IOR was first described by Posner and Cohen (1984) as the inhibition of shifting attention back to a previously inspected location. These authors observed that presenting a peripheral cue at the position of the target facilitated discrimination of the target with a brief cuetarget onset asynchrony (CTOA) but hampers target discrimination with longer CTOAs. Therefore, IOR refers to better performance for targets at a different position than the cue than for targets at the same position. This result is typical of cues that are not predictive of the target's position.

Posner and Cohen (1984) speculated that the cue initially captured attention, leading to faster discrimination with a short CTOA when the cue was presented at the same position as the target than when it was presented at a different position. After some time, however, participants withdraw their attention if no relevant target is presented at the cued location. According to Posner and Cohen, the participants use some of the long CTOA to even inhibit the return of attention to the previously inspected location (therefore the name IOR). Subsequent research revealed that components besides attention capture, such as motor inhibition and sensory habituation, can also contribute to IOR (for reviews, see Klein, 2000; Lupiàñez, 2010; Wang \& Klein, 2010).

Top-down control: Contingent attentional capture

Importantly, in the present context, IOR also features prominently in one of the large debates in attention research: the question of the extent to which attention capture is top-down contingent (cf. Folk, Remington, \& Johnston, 1992) versus stimulus-driven (or bottom-up; cf. Theeuwes, 1992, 2010). 
According to Folk et al., only cues with a relevant feature that matches the searched-for target feature capture attention. Folk et al. based their conclusion on experiments with two sorts of nonpredictive cues. They used either one colored (red) cue as a color-singleton together with three white cues, or a single abrupt-onset cue. Both cue types were presented with the same CTOA before the relevant target and did not predict the target position. Critically, in one block, the participants searched only for abrupt-onset targets, whereas in the other block, they searched only for color-defined (red) target singletons. In line with Folk et al.'s conception of top-down contingent capture, only the cue with a feature matching the top-down set captured attention. If the participants searched for onset targets, only the onset cue captured attention, but the color cue failed to capture attention. This pattern of results was reversed when the participants searched for a color-defined target: Here, the color-singleton cue captured attention but the abrupt-onset cue did not. Later research showed that even the cue's color has to resemble and match the set of searched-for target colors (cf. Anderson \& Folk, 2010; Folk \& Remington, 1998; Lamy, Leber, \& Egeth, 2004). If the participants search for red targets, a red cue but not a green cue captures attention but if the participants search for green targets, a green cue but not a red cue captures attention (e.g., Ansorge, Kiss, Worschech, \& Eimer, 2011; Eimer \& Kiss, 2008; Worschech \& Ansorge, 2012; for a review, see Burnham, 2007).

\section{Bottom-up control: Stimulus-driven capture}

In contrast to the top-down contingent capture theory, according to Theeuwes (2010), any strong feature contrast (e.g., color contrast) captures attention (e.g., Itti, Koch, \& Niebur, 1998; Schreij, Theeuwes, \& Olivers, 2010a). For example, Schreij et al. (2010a) adapted the experimental protocol of the colorsearch conditions of Folk et al. (1992) by presenting an additional abrupt-onset singleton briefly before the target (Schreij, Owens, \& Theeuwes, 2008; Schreij et al., 2010a). These authors observed that the abrupt-onset singleton delayed the discrimination of the color-defined target relative to a condition without an additional onset distractor. Schreij et al. (2010a) argued that the abrupt onset must have captured attention although it was completely task-irrelevant and did not match the searched-for property. Moreover if the abrupt-onset singleton was presented with a long CTOA of $900 \mathrm{~ms}$ before the color-defined target, interference disappeared just as if attention had been deallocated from the abrupt-onset distractor (Schreij, Theeuwes, \& Olivers, 2010b).

\section{Attentional deallocation}

This latter observation supports Theeuwes, Atchley, and Kramer's (2000) more general argument that the absence of capture with nonmatching cues that was typically found in contingent-capture experiments à la Folk et al. reflected swift deallocation of attention after initial capture. To understand this, remember that the cues in the contingent-capture studies (e.g., Folk et al., 1992) were usually nonpredictive of the target position. Therefore, there was no incentive for the participants to maintain attention at a cued location and deallocation of attention away from the cues was encouraged. In addition, according to Theeuwes et al., the brief CTOA of $150 \mathrm{~ms}$ that is commonly used in contingent-capture experiments (cf. Folk et al., 1992) is already long enough for (1) attention capture by matching and nonmatching cues and (2) for deallocation only after the nonmatching cues. With the matching cues, deallocation is delayed because a higher cuetarget resemblance makes cue recognition more difficult. As a consequence, traces of capture can still be seen with a short enough CTOA in top-down matching conditions but no longer in the nonmatching conditions.

\section{Attentional deallocation: Evidence from IOR}

When this deallocation hypothesis was tested, the results were not always in line with it (e.g., Ansorge et al., 2011; Gibson \& Amelio, 2000; Remington, Folk, \& McLean, 2001; Worschech \& Ansorge, 2012). Of particular relevance with respect to IOR, Gibson and Amelio (2000) used long CTOAs of $1 \mathrm{~s}$ in the protocol of Folk et al. (1992) but IOR was neither observed after matching nor after nonmatching color-singleton cues (for the latter result, see also Pratt \& McAuliffe, 2002). Gibson and Amelio only found deallocation in the form of IOR after abruptonset cues. In addition, IOR was only found for abrupt-onset cues followed by abrupt-onset targets (i.e., matching the topdown set). By contrast, there was no IOR after abrupt-onset cues followed by color targets.

Jointly, these findings are in disagreement with Theeuwes et al. (2000; Theeuwes, 2010) because the deallocation explanation predicts IOR after attention capture by nonmatching cues that should have an earlier onset than after matching cues. Remember that deallocation after matching cues was assumed to trail behind deallocation after the nonmatching cues. Therefore, until today researchers dispute the deallocation hypothesis (cf. Lamy, 2010).

\section{Purpose of the present study}

These conflicting results were the point of departure for the present study. We wanted to test two critical predictions of Theeuwes et al.'s (2000) deallocation explanation. We aimed at testing whether IOR is found after color singletons and we wanted to test the further question whether IOR is stronger after nonmatching (or irrelevant) color-singleton cues than after matching (or relevant) color-singleton cues. Evidently, the failure to observe the predicted pattern in prior research 
(cf. Gibson \& Amelio, 2000) necessitated some procedural changes.

In comparison to previous studies, we took two measures to more thoroughly investigate IOR. First, in Experiments 1, 2, and 5, we used saccadic IOR (Abrams \& Dobkin, 1994; Dorris, Klein, Everling, \& Munoz, 2002; Taylor \& Klein, 1998) because manual responses are not necessarily the most sensitive measure of attention and IOR (Briand, Larrison, \& Sereno, 2000; Cole, Kuhn, Heywood, \& Kentridge, 2009; Deubel, 2008; Deubel \& Schneider, 1996). Second, we studied IOR as a function of response times (RTs). To that end, we vincentized response latencies (with mean RTs for quintiles of the response distribution, from fast to slow responses) (van Zoest, Donk, \& Theeuwes, 2004). Since deallocation and IOR are a function of time since cue onset, IOR can be overlooked if one only considers the total mean RT, and we expected more IOR with slower than faster responses.

\section{The general procedure}

In the valid condition, the cue was presented at the same position as the subsequent saccade target. In the invalid condition, the cue was presented at a different position than the target. Because the cue did not predict the saccade target position, deallocation and IOR were encouraged. Saccadic IOR should be reflected in faster responses in invalid than valid conditions.

In the relevant-cue trials of Experiments 1 to 3 , one predefined color cue (e.g., green) told the participants to keep the cue position in mind. This was necessary for the later discrimination of a second target at the cued position. This discrimination target was presented after the saccade target, at the end of the trial (see Fig. 1). In the irrelevant-cue trials, participants had to ignore the cue, because no discrimination was required at the end of the trial. Relevant and irrelevant cues had different fixed, known colors. For example, if the relevant cue was green, the irrelevant cue was blue. Therefore, participants were enabled to set up top-down settings for search of the relevant and for disregarding the irrelevant colors.

The relevant and irrelevant cues thus corresponded to topdown matching and nonmatching color cues in past contingentcapture studies, in so far as the relevant cue matched and the irrelevant cue did not match the search settings. However, the relevant and irrelevant cues differed from the top-down matching cues and nonmatching cues in previous research, in so far as the relevant cues were necessary to find the discrimination target: Participants had to keep the relevant cue in short-term memory in order to locate the discrimination target. This had not been the case in past contingent-capture studies. We will get back to this difference in the discussion of Experiment 1 and in the General Discussion. To make this distinction in procedures clear, we will use the terms "relevant" and "irrelevant" in Experiments 1-3. We will only use the terms "matching" and "nonmatching" in

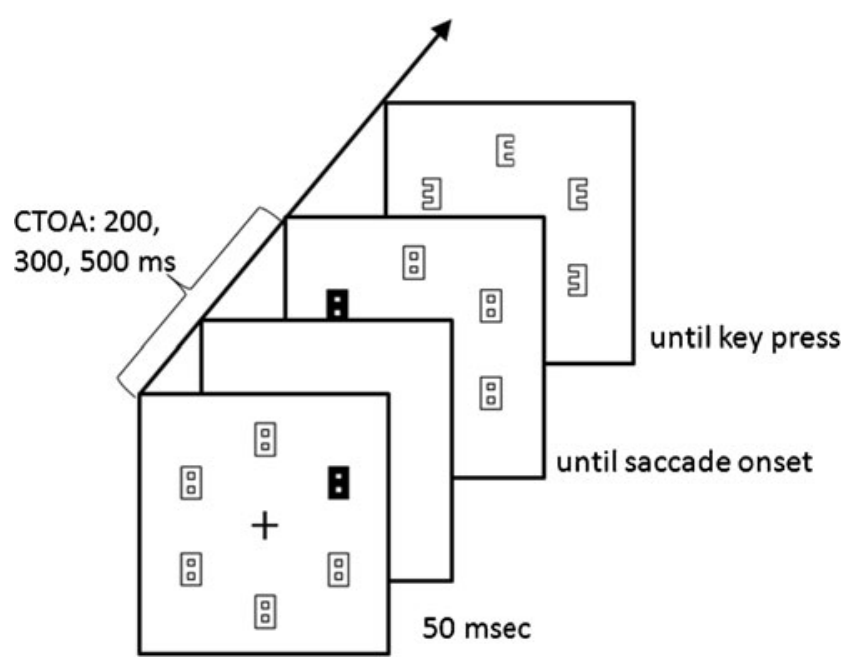

Fig. 1 Depicted is an example of an invalid trial. The first (lower) display was a cueing display, in which a cue (illustrated as a black figure-8) was presented in green or blue, with one color indicating the relevant and the other the irrelevant cue. The second (middle) display was a saccade-target display, in which a saccade target (illustrated as a black figure-8) was shown in red (in Exps. 1 and 3) or in the color of the relevant cue (in Exp. 2). The third (upper) display was a discrimination-target display, in which a discrimination target was shown. The discrimination-target display was only shown after the relevant cue. The discrimination target was presented at the relevant cue's position. The arrow depicts the direction of time. Stimuli are not drawn to scale. $\mathrm{CTOA}=$ cue-target onset asynchrony: $500 \mathrm{~ms}$ in Experiment 1, $300 \mathrm{~ms}$ in Experiments 1 and 3, and $200 \mathrm{~ms}$ in Experiments 1 and 2

Experiments 4 and 5, because in these experiments the cues were truly unrelated to the task.

\section{Experiment 1}

Participants made saccades to colored saccade targets. In addition, only after relevant cues, participants had to subsequently identify a discrimination target. Cues and targets were of different fixed colors. For example, the saccade target was red, the relevant cue blue, and the irrelevant cue green. These colors were known to the participants.

These were our predictions. First, because all cues did not inform about the saccade target positions and because saccade programming requires shifting of attention to the saccade target (Deubel \& Schneider, 1996), we expected saccadic IOR. Second, saccadic IOR should at least be present among the slower responses after vincentization of response times. Third, between participants, we varied the CTOA. The CTOA was 500,300 , or $200 \mathrm{~ms}$. In this way, we were able to test whether saccadic IOR increases (and capture decreases) with CTOA (cf. Posner \& Cohen, 1984). Also, overall net attention capture rather than saccadic IOR might be found with a short CTOA. Fourth, if the deallocation theory holds true, we expected stronger or earlier IOR to irrelevant than relevant cues. An earlier onset of IOR should be reflected in IOR 
among shorter RTs in irrelevant than relevant cueing conditions. Especially in the short CTOA, we might observe capture with relevant cues but saccadic IOR with irrelevant cues. Finally, with the relevant cue, we expected to see overall stronger capture effects.

\section{Method}

Participants The groups consisted of ten (five male, five female), 14 (four male, ten female), and ten (five male, five female) participants - mostly students, with mean ages of 25 , 23, and 21 years - in the 500-, 300-, and 200-ms CTOA conditions, respectively. All participants reported normal or corrected-to-normal visual acuity and received course credit in exchange for their participation.

Apparatus Visual stimuli were presented on a 19-in. CRT color monitor (Sony Multiscan G400) with a screen resolution of $1,024 \times 768$ pixels. Its refresh rate was $100 \mathrm{~Hz}$. The participants sat at a distance of $57 \mathrm{~cm}$ from the screen in a quiet, dimly lit room, with their head resting in a chinrest to ensure a constant viewing distance and a straight-ahead gaze direction. Manual responses to the discrimination targets were registered via a standard keyboard, placed directly in front of the observers. Participants' manual responses were given by the keys \#F and \#J (labeled "left" and "right"). Saccades were recorded with an EyeLink 1000 Desktop Mount system (SR Research, Mississauga, Ontario, Canada) with a $35-\mathrm{mm}$ lens, and EyeLink Software version 4.52, sampling at maximal resolution. Eyetracking was monocular from the dominant eye. A 9-point calibration was used to adjust the eyetracker in advance of every single block.

Stimuli See also Fig. 1. All stimuli were lighter $\left(\sim 30 \mathrm{~cd} / \mathrm{m}^{2}\right)$ than the dark background $\left(\sim 0.2 \mathrm{~cd} / \mathrm{m}^{2}\right)$. The stimuli in the cue and saccade-target displays consisted of six digital figure- $8 \mathrm{~s}$ of a size of $1.7^{\circ} \times 1.0^{\circ}$, with a stroke width of $0.3^{\circ}$. The figure- $8 \mathrm{~s}$ were positioned $0^{\circ}, 60^{\circ}, 120^{\circ}, 180^{\circ}, 240^{\circ}$, and $300^{\circ}$ from the vertical meridian on a virtual circle around the center, with an eccentricity of $7.0^{\circ}$. The top-most and lowest position were only used as filler positions and never contained the cue or the target. The color singleton in the cue display was either green (CIE color coordinates: -30.2, 24.9) or blue (CIE: 46.9, -89.0). During the entire experimental session, one of these colors was relevant and the other irrelevant. The saccade target color singleton was red (CIE: 47.6, 41.1). All other stimuli were gray (CIE: 6.9, 16.8). In addition to the cue and target displays, a target-discrimination display was shown after the relevant cues ( $50 \%$ of all trials). To create distinct discrimination forms, one vertical line of each of the six figure- $8 \mathrm{~s}$ was deleted, so that three digital letter-Es and three digital figure-3s replaced the six figure-8s. In the discrimination-target display, all of the stimuli were gray.
Procedure At the beginning of every trial, a fixation cross $\left(0.7^{\circ}\right.$ $\times 0.7^{\circ}$ ) was shown in the center of the screen. Participants started by looking at the fixation cross and pressing the space bar. At this time, a drift correction of the eyetracking signal was conducted if the currently measured eye position deviated by more than $1.5^{\circ}$ of the previous trial's fixation at screen center. In addition, the experimenter visually monitored eye position. Next, the fixation display persisted for $500 \mathrm{~ms}$, and then the cueing display was shown for $50 \mathrm{~ms}$. After the CTOA, the saccade target display was shown and observers made a saccade to the red color singleton. The CTOA varied between participants from 500 to $200 \mathrm{~ms}$. The saccade target display was shown until saccade onset or until $1 \mathrm{~s}$ had elapsed.

In the valid trials, the saccade target was at the cued position. In the invalid trials, the saccade target was at an uncued position. Because saccade target positions and cue positions were uncorrelated, valid trials made up $25 \%$ and invalid trials $75 \%$ of all trials.

In the relevant-cue trials, the color cue indicated the position of the discrimination target in the final discrimination-target display. Participants therefore had to attend to the cue's position and keep it in mind for later retrieval of the position of the discrimination target. The participants signaled the identity of the discrimination target by a keypress. In the irrelevant-cue trials, the color cue could be ignored. No discrimination target was shown, and no manual response was required. Relevant and irrelevant cues had different colors (green and blue, respectively), and participants were informed that color was relevant.

The experiment consisted of one training block and four data acquisition blocks. Every block consisted of 64 trials. Every block thus corresponded to one repetition of each of the 2 cue types (relevant; irrelevant) $\times 4$ cue positions $\times 4$ saccade-target positions. Within blocks, different conditions were realized in a pseudorandom order. Between blocks, participants were encouraged to take short breaks.

Written feedback was given after early saccades (saccadic RT $<100 \mathrm{~ms}$ ) and late saccades (saccadic RT $>1 \mathrm{~s}$ ). Thus, a relatively broad distribution of saccadic RTs was allowed. This was done for the sake of more information on the development of saccadic IOR over time. In addition, in relevant-cue trials of the training block, written feedback indicated whether the actual target discrimination response was correct. In the experimental trials, no feedback about the discrimination response was given.

\section{Results and discussion}

Capture and IOR were calculated as the difference between valid and invalid conditions. Thus, positive values reflect saccadic IOR and negative values reflect capture. If Mauchly tests indicated a violation of the sphericity assumption, the degrees of freedom were corrected by Greenhouse-Geisser's $\varepsilon$ and the corrected alpha level is reported. 
Saccade landing position was calculated as the $x-y$ coordinates of the eyetracker signal at the time at which eye velocity returned to a presaccadic baseline level. A saccade counted as correct if it landed in an area of $1.5^{\circ}$ around the center of the saccade target. A saccade counted as erroneous if the eyes landed in an area of $1.5^{\circ}$ around the center of a distractor. In Experiment 1, $3.4 \%$ were discarded because they were faster than $100 \mathrm{~ms}$.

Saccadic RTs Saccadic RTs were calculated as the times between (1) the onset of the saccade-target stimulus and (2) the time of a local velocity minimum that immediately preceded the point in time at which eye velocity exceeded $80 \%$ s. The results can be seen in Fig. 2.

A mixed-model analysis of variance (ANOVA) ${ }^{1}$ of the correct saccadic RTs was run, with the repeated-measures variables of validity (valid; invalid), cue relevance (relevant cue; irrelevant cue), and quintile (1st to 5 th), and the betweenparticipants variable CTOA (200, 300, or $500 \mathrm{~ms})$. All main effects became significant. The validity effect, $F(1,31)=$ 25.23, $p<.01$, reflected IOR. That is, saccadic RTs were slower in valid (260-ms) than invalid (229-ms) conditions. The effect of relevance, $F(1,31)=8.19, p<.01$, was due to faster saccadic RTs with irrelevant cues $(241 \mathrm{~ms})$ than with relevant cues $(248 \mathrm{~ms})$, indicating a dual-task cost when the cue location had to be kept in memory. The effect of CTOA, $F(2,31)=4.85, p<.05$, indicated that saccadic $\mathrm{RT}$ was lower with a CTOA of $500 \mathrm{~ms}(218 \mathrm{~ms})$ than with a CTOA of $300 \mathrm{~ms}$ ( $283 \mathrm{~ms} ; p<.05$, Bonferroni corrected) but that there were no significant differences between CTOA $200 \mathrm{~ms}$ $(232 \mathrm{~ms}$ ) and the other CTOAs (both $p \mathrm{~s}>.09$ ). The faster RTs with the long CTOA may be due to increasing response preparation. There was also a trivial main effect of quintile, $F(4,124)=105.97, p<.01$, reflecting increasing saccadic RTs across quintiles. This main effect will not be reported in subsequent experiments.

There was evidence that IOR increased for slower responses: The significant interaction between validity and quintile, $F(4$, $124)=24.14, p<.01$, showed that saccadic IOR increased with increasing saccadic RT. Capture amounted to a nonsignificant $-1 \mathrm{~ms}, t(33)<1.00$, in the 1st quintile, and significant saccadic IOR of $37,37,47$, and $73 \mathrm{~ms}$, was found in the 2nd to 5th quintile, respectively, all $p \mathrm{~s}>2.60$, all $p \mathrm{~s}<.05$.

Furthermore, we found two significant interactions that do not directly relate to our hypothesis: The interaction between quintile and CTOA, $F(8,124)=3.78, p<.05$, resulted from stronger increases of RTs over time with a CTOA of $300 \mathrm{~ms}$ than with CTOAs of $500 \mathrm{~ms}$ and $200 \mathrm{~ms}$. The three-way interaction of relevance, quintile, and CTOA, $F(8,124)=$

\footnotetext{
${ }^{1}$ Complementary ANOVAs were conducted in Experiments 1-3 with only those trials of the relevant-cue condition in which the discrimination target was correctly discriminated. This led to the elimination of less than $2 \%$ of the data. The results of these ANOVAs were very similar to those in the main text and are not reported separately.
}

$4.11, p<.05$, was due to stronger increases in the relevant conditions of the $300-\mathrm{ms}$ as compared to the $200-\mathrm{ms}$ CTOA.

Furthermore, two of the interactions only approached significance, and only the first of these was relevant for our hypothesis. The significant interaction of validity and relevance, $F(1,31)=3.04, p=.09$, showed that saccadic IOR tended to be larger with irrelevant $(36 \mathrm{~ms})$ than with relevant (28 ms) cues, as claimed by the deallocation theory. In addition, the interaction of relevance by quintile, $F(1,124)=2.69$, $p=.09$, showed that delays of saccadic RT with relevant as compared to irrelevant cues were absent among the fastest responses (1st quintile: relevant $\mathrm{RT}=172$, irrelevant $\mathrm{RT}=$ 171) and increased with increasing RT (e.g., 5th quintile: relevant $\mathrm{RT}=359 \mathrm{~ms}$, irrelevant $\mathrm{RT}=345 \mathrm{~ms}$ ).

Otherwise, we observed no significant interactions, all $F_{\mathrm{S}}<2.40$, all $p \mathrm{~s}>.10$, and especially no three-way interaction of Validity $\times$ Relevance $\times$ Quintile, $F<1.00$.

Erroneous saccades Separately for each CTOA, arc-sine transformed rates of erroneous saccades between $100 \mathrm{~ms}$ and $1 \mathrm{~s}$ were computed to compensate for the correlation of mean and variance with probability values (Sokal \& Rohlf, 1981). These data were analyzed as a function of the cue's relevance (relevant vs. irrelevant) and of the saccade's direction (to the cued position vs. away from the cued position). Only invalid trials were considered. ${ }^{2}$

We analyzed the error rates in order to rule out speedaccuracy trade-offs with saccadic RT. In a mixed-model ANOVA, with the repeated-measures variables relevance and saccade direction, and the between-participants variable CTOA, the main effect of CTOA failed, $F(2,31)=2.88, p=$ .07 , but the two other main effects, both $F_{\mathrm{s}}(1,31)>28.00$, both $p \mathrm{~s}<.01$, and all interactions, all $F_{\mathrm{S}}>5.30$, all $p \mathrm{~s}<.05$, became significant. To understand the interactions, follow-up ANOVAs were conducted with data split up for different CTOAs.

With a CTOA of $500 \mathrm{~ms}$, there were no significant main effects or interactions, $F \mathrm{~s}<3.20, p \mathrm{~s}>.10$. The overall error rate was $2.4 \%$. With shorter CTOAs of $300 \mathrm{~ms}$ and $200 \mathrm{~ms}$, follow-up ANOVAs led to significant main effects of cue relevance, $F_{\mathrm{s}}>10.00, p \mathrm{~s}<.01$, and of direction, $F_{\mathrm{s}}>9.00$, $p \mathrm{~s}<.05$, as well as to significant interactions, $F_{\mathrm{S}}>9.00, p<.01$. There were more erroneous saccades in the relevant than the irrelevant conditions (CTOA of $300 \mathrm{~ms}: 5.5 \%$ vs. $2.2 \%$; CTOA of $200 \mathrm{~ms}: 12.3 \%$ vs. $4.6 \%$ ). There were also more erroneous saccades towards the cue than away from it (CTOA of $300 \mathrm{~ms}$ : $6.7 \%$ vs. $1.0 \%$; CTOA of $200 \mathrm{~ms}: 15.2 \%$ vs. $1.7 \%$ ). Erroneous

\footnotetext{
${ }^{2}$ Per definition, erroneous saccades were directed away from the cue in the valid condition $(1.3 \%, 1.6 \%$ in Exps. 1 and 2, respectively). To understand whether capture (toward the cue) and IOR (away from the cue) were found in the rates of erroneous saccades, we therefore compared the rates of the erroneous saccades toward the cue and away from the cue in the invalid condition only.
} 
saccades to the cued location were more frequent for the relevant cues than for the irrelevant cues (difference between saccades toward the cue and away from the cues: CTOA of $300 \mathrm{~ms}: 9.0 \%$ vs. $2.5 \%$; CTOA of $200 \mathrm{~ms}: 20.2 \%$ vs. $6.7 \%$ ). Overall, there is no evidence for speed-accuracy trade-offs between accuracy and saccadic RTs.

\section{Discussion}

CTOAs ranging from 500 to $200 \mathrm{~ms}$ showed strong saccadic IOR to color-singleton cues. Consistent with our predictions,
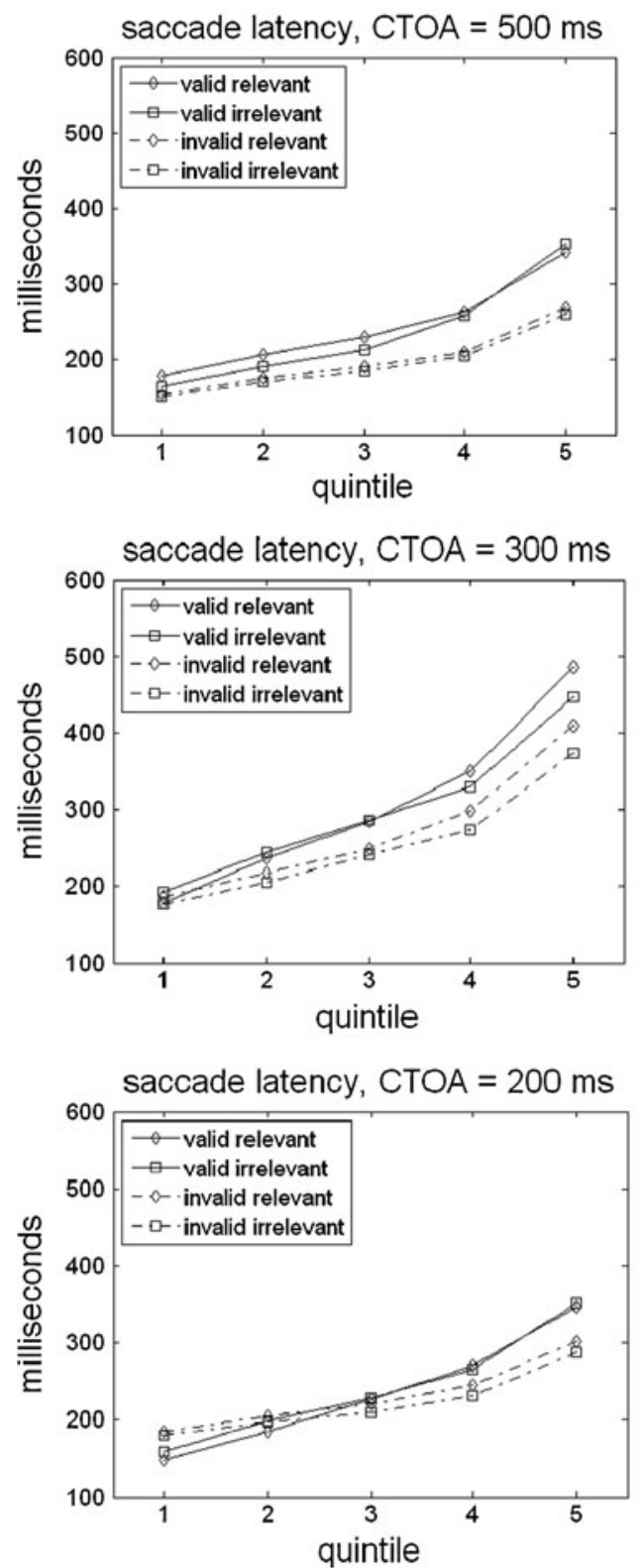

Fig. 2 Experiment 1: On the left, saccadic latencies (in milliseconds) as a function of the cue's relevance, validity, and the quintile of the saccadic latency distribution. On the right, we plotted the saccadic latencies in saccadic IOR was greater for slower responses. Unexpectedly, we did not observe that CTOA affected saccadic IOR. IOR did not increase with longer CTOAs, which may be due to the restricted range of CTOAs used (200 to $500 \mathrm{~ms}$ ). Perhaps longer CTOAs (i.e., $1000 \mathrm{~ms}$ ) would produce greater IOR. Furthermore, we found only a nonsignificant tendency for relevance to affect saccadic IOR. We had expected stronger and earlier saccadic IOR in irrelevant than relevant conditions because attention can be more rapidly disengaged when cues are irrelevant. The present study does not provide strong support for this claim.
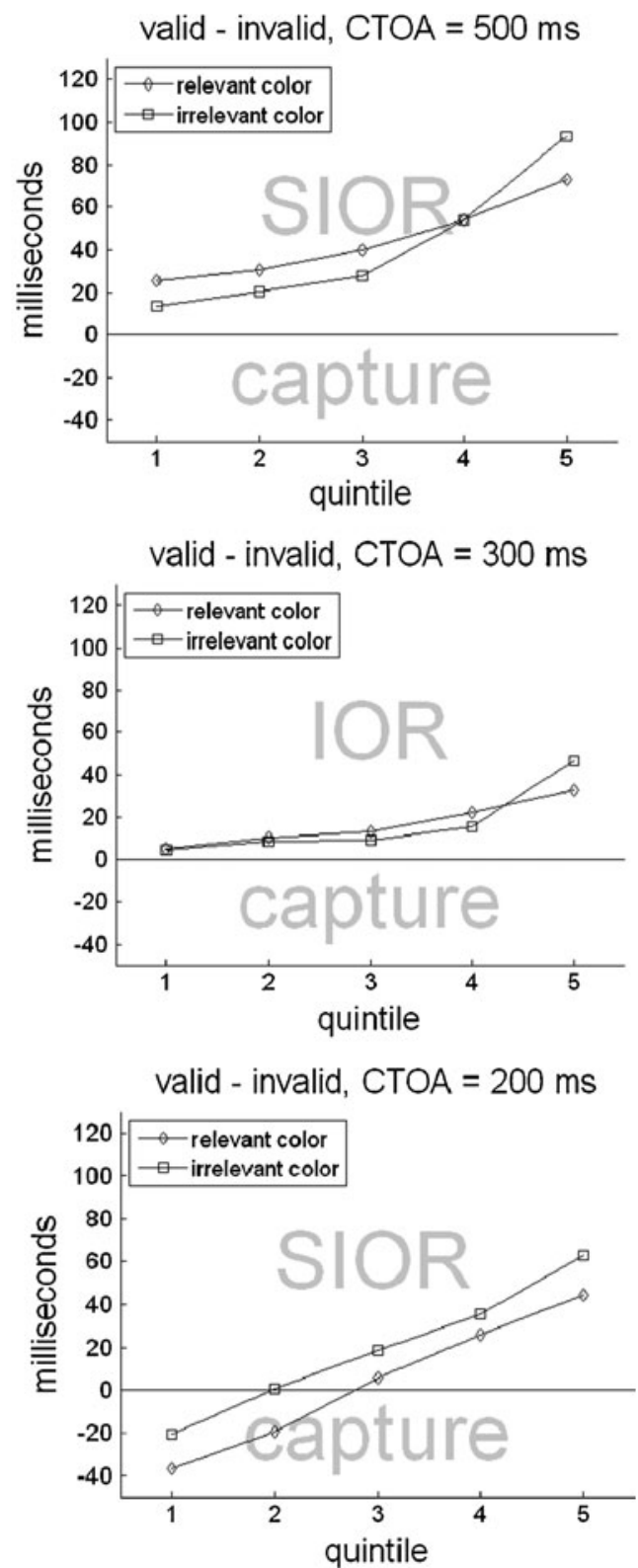

valid minus invalid conditions. Inhibition of return (SIOR) results in positive values, and attentional capture in negative values. The data from Experiment 1's different CTOA conditions are shown in different rows 
However, the presence of saccadic IOR with color singletons confirms that deallocation also takes place after color singleton cues (cf. Godijn \& Theeuwes, 2004; Kim \& Cave, 1999). This result is at variance with the studies showing no deallocation and no IOR to color-singleton cues (cf. Gibson \& Amelio, 2000; Remington et al., 2001). One important difference between the present study and past research concerned our use of saccades. Because saccades are more sensitive to IOR than manual responses (e.g., Zhang \& Zhang, 2011; see also Godijn \& Theeuwes, 2004), less IOR to color singletons in past studies could have been due to the use of manual responses. We will address this question in experiments reported below.

A second difference between the present and past studies concerned our use of a memory task: Participants had to keep the relevant cue's position in memory. This was reflected in RT costs with relevant cues as compared to irrelevant cues. Costs were greater with longer RT. Our task is different from standard contingent-capture experiments in which participants ignored all cues. Importantly, past studies showed that keeping a location in memory affects attention (Awh, Vogel, \& Oh, 2006) and saccades (Belopolsky \& Theeuwes, 2009). Most critically, keeping a location in memory can create interference with saccade execution (e.g., Belopolsky \& Theeuwes, 2011). We think this is an unlikely origin of the IOR effect in the present study because participants only had to keep the relevant, but not the irrelevant cue in memory. Compliance with this instruction was reflected in shorter saccadic RTs with irrelevant than relevant cues. If the memory trace was weaker with irrelevant than relevant cues, interference and IOR should have been smaller with irrelevant than relevant cues. Yet the opposite was found. IOR was numerically, but not significantly, stronger with irrelevant than relevant cues.

\section{Experiment 2}

In Experiment 2, we wished to follow up on the nonsignificant tendency for greater saccadic IOR to irrelevant than relevant cues. This difference is predicted by deallocation theory because irrelevant cues allow for more rapid disengagement than relevant cues. Also, deallocation theory predicts an earlier onset of IOR with irrelevant than relevant cues, which was not observed in Experiment 1. We therefore refined the protocol in Experiment 2 to provide a more sensitive test of these hypotheses. Past research indicated that participants can have difficulties searching for two colors, and that participants can optimally set up their search settings for only one color at a time (Ansorge \& Horstmann, 2007; Folk \& Anderson, 2010; Grubert \& Eimer, 2013). In Experiment 2, we therefore used the same color for the relevant cue and the saccade target. That is, the color of a relevant cue matched that of the target, whereas the color of an irrelevant cue did not. Under these conditions, participants should show less saccadic IOR and more capture to relevant/matching than to irrelevant/nonmatching cues. Also, in irrelevant/nonmatching conditions, saccadic IOR should start earlier (among shorter RTs).

\section{Method}

Participants Thirteen (three male, ten female) participants, mostly students, with a mean age of 23 years participated. All participants reported normal or corrected-to-normal visual acuity and received course credit for their participation.

Apparatus, stimuli, and procedure These were the same as in Experiment 1's 200-ms CTOA condition, with the exception that the relevant cue and the saccade target were of the same color.

Results

Of all responses, $4.1 \%$ were faster than $100 \mathrm{~ms}$. These were discarded.

Saccadic RTs Correct saccadic RTs were submitted to a repeated-measures ANOVA, with the within-participant variables cue relevance (relevant cue; irrelevant cue), validity (valid; invalid), and quintile (1st to 5th).

Looking at Fig. 3, one can see that among the fast responses, capture occurred with relevant cues, whereas saccadic IOR was observed with irrelevant cues. This impression was supported by ANOVA. Importantly, we found a significant threeway interaction, $F(1,12)=4.65, p<.05$, reflecting capture with relevant cues in the $1 \mathrm{st}$ and 2 nd quintile $[-46 \mathrm{~ms}$ and $-28 \mathrm{~ms}$, respectively, both $t \mathrm{~s}(12)<-2$, both $p \mathrm{~s}<.05$ ], but saccadic IOR with irrelevant cues $[15 \mathrm{~ms}, t(12)=1.32, p=.21$, and $46 \mathrm{~ms}$, respectively, $t(12)=3.97, p<.01]$. By contrast, among the slower RTs, saccadic IOR was found with both relevant [3rd to 5th quintiles: $9 \mathrm{~ms}, t<1.00,77 \mathrm{~ms}, t(12)=1.90, p=.08$, and $124 \mathrm{~ms}$, $t(12)=2.94, p<.05]$ and irrelevant [3rd to 5th quintiles: $74 \mathrm{~ms}$, $104 \mathrm{~ms}$, and $85 \mathrm{~ms}$, all $t \mathrm{~s}(12)>4.40$, all $p \mathrm{~s}<.01]$ cues.

We also found a significant effect of validity, $F(1,12)=$ $8.95, p<.05$, an interaction of validity and quintile, $F(1,12)=$ $20.85, p<.01$, and an almost significant interaction of relevance and quintile, $F(1,12)=4.18, p=.051$. The validity effect reflected saccadic IOR, with slower saccadic RTs in valid (337-ms) than invalid (290-ms) conditions. The interactions between relevance and quintile, and between position and quintile demonstrated that saccadic IOR and the relevance effect both built up over time. The main effect of relevance, $F<$ 1.00 , and the interaction of relevance and position, $F(1,12)=$ $3.12, p=.10$, were not significant.

Erroneous saccades ${ }^{2}$ A repeated measures ANOVA of the arcsine-transformed rates of erroneous saccades in the invalid 


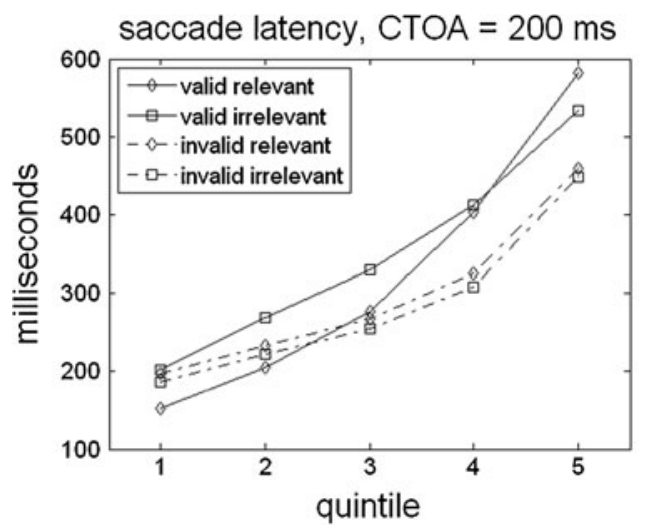

Fig. 3 Experiment 2: On the left are saccadic latencies (in milliseconds) as a function of the cue's relevance, validity, and the quintile of the saccadic latency distribution. On the right, we plotted the saccadic

conditions, with the two variables of cue relevance (relevant cue; irrelevant cue) and of saccade direction (toward the cue; away from the cue) on the data from the invalid condition led to significant effects of cue relevance, $F(1,12)=9.01, p<.05$, and direction, $F(1,12)=11.95, p<.01$, and a significant interaction, $F(1,12)=9.58, p<.01$. Erroneous saccades were more likely in the relevant $(8.3 \%)$ than the irrelevant (3.4\%) condition. The rate of the erroneous saccades toward the cued location $(9.0 \%)$ exceeded that away from the cue $(2.7 \%)$. Also, erroneous saccades to the cues were more frequent with the relevant cues $[10.9 \% ; t(12)=3.66, p<.01]$ than with the irrelevant cues $[1.8 \% ; t(12)=1.16, p=.30]$. Overall, there was no evidence for speed-accuracy trade-off.

\section{Discussion}

In Experiment 2, the relevant cue color matched the target color as in typical contingent capture paradigms. The threeway interaction between quintile, validity, and cue relevance showed that saccadic IOR occurred earlier with irrelevant than relevant cues. This finding is in line with Theeuwes et al.'s (2000) deallocation theory. In addition, attentional capture was found for the relevant cues among the fastest responses. Jointly, the data thus suggested the existence of both more saccadic IOR to irrelevant cues (Theeuwes et al., 2000) and more contingent capture to relevant cues (Folk et al., 1992).

Intertrial priming might have contributed to the capture effect that we ascribed to contingent capture. Some researchers noted that finding the target by one specific color (e.g., red) in trial $n$ primes capture by the same color in the immediately following trial $n+1$ (Maljkovic \& Nakayama, 1994). Previous studies have ruled out intertrial priming as the sole origin of capture, and confirmed the contingent capture effect (Ansorge \& Horstmann, 2007; Irons, Folk, \& Remington, 2012; Worschech \& Ansorge, 2012). In these studies, two

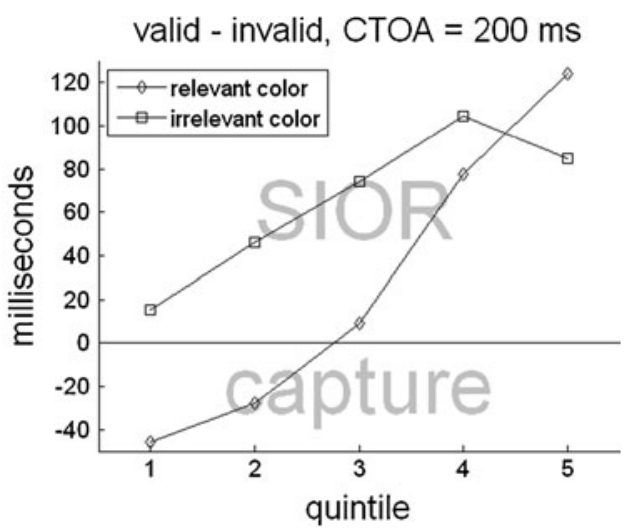

latencies in valid minus invalid conditions. Saccadic inhibition of return (SIOR) results in positive values, and attentional capture in negative values

relevant target colors (e.g., red and green) were used, and capture by one relevant color cue (e.g., red) was stronger than capture by an irrelevant color cue (e.g., blue) even if the alternative relevant color (e.g., green) had been used for the target in the preceding trial. In the present experiment with only a single relevant color, this analysis cannot be conducted. Therefore, it is possible that inter-trial priming contributed to the present capture effect.

\section{Experiment 3}

In Experiment 3, we tested whether deallocation can also be found with manual responses. To that end, a manual pointing response to the targets instead of a saccadic response was required. We used a CTOA of $300 \mathrm{~ms}$ because $200 \mathrm{~ms}$ seemed possibly too short for manual responses to lead to IOR at all (Klein, 2000; Taylor \& Klein, 1998). Otherwise conditions were very similar to Experiment 1.

\section{Method}

Participants A group of 21 (seven male, 14 female) students, with a mean age of 21 years participated. They had normal or corrected-to-normal visual acuity. They received course credit in exchange for their participation.

Apparatus, stimuli, and procedure A 19-in. Ilyama ProLite T1931SR touchscreen was used. It was slanted (with the upper edge away from the participants by $\sim 80^{\circ}$ ) so that the participants looked down on the screen and could conveniently move their fingers across the screen. A pointing-target display was used in which participants had to lift their right index finger from the cross at screen center and had to put it on the pointing target as quickly and accurately as possible. A response counted as correct if the right index finger landed in a 
target area of $90 \times 120$ pixels (corresponding to a rectangle with a $1.0^{\circ}$ frame around the pointing target of $30 \times 90$ pixels). Between trials, the participants had to put their right index finger in the center of the screen.

With relevant cues, target discrimination was required. Participants had to press one of two "buttons" displayed on the touchscreen, one with a digital $\mathrm{E}$, the other one with a digital 3 on it. The buttons were in the lower left and right corners of the screen. No keyboard was used and eye movements were not tracked. Otherwise the experiment was very similar to Experiment 1.

\section{Results}

Out of all responses, $5.9 \%$ were discarded as anticipations (with RT $<100 \mathrm{~ms}$ ). Another three responses were discarded because they were wrongly directed toward a cued distractor. Two of these errors went to a relevantly cued distractor and one to an irrelevantly cued distractor.

Pointing RTs For the results, see also Fig. 4. The correct RTs were submitted to a three-way ANOVA, with the same withinparticipants variables that had been used in the preceding experiments, cue type (relevant cue, irrelevant cue), validity (valid, invalid), and quintile. The ANOVA revealed a significant main effect of validity, $F(1,20)=8.42, p<.01$, a significant interaction of validity and quintile, $F(4,80)=$ $4.54, p<.05$, and an almost significant interaction of relevance and quintile, $F(4,80)=3.23, p=.07$. The main effect of validity reflected that valid RTs were delayed $15 \mathrm{~ms}$, as compared to invalid RTs ( 245 vs. $230 \mathrm{~ms}$ ). The interaction of validity and quintile showed that IOR increased with increasing RTs, with IOR amounting to 5, 9, 11, 19, and $40 \mathrm{~ms}$, from the 1 st to the 5 th quintile. A numerical increase of the dual-task costs (calculated as irrelevant RT minus relevant RT) with an increasing RT accounted for the tendency toward a significant interaction between relevance and quintile, 1 st to 5 th quintile: $6,2,-3,-7$, and $-19 \mathrm{~ms}$. The other main effects and interactions were not significant, $F_{\mathrm{S}}<1.70, p \mathrm{~s}>.20$.

Movement times The ANOVA of the movement times (from lifting until landing) led to a main effect of relevance, $F(1,20)=$ 45.41, $p<.01$, and a significant interaction between relevance and quintile, $F(4,80)=23.55, p<.01$. The effect of relevance was caused by slower RTs with relevant than with irrelevant cues (663 vs. $627 \mathrm{~ms}$ ). The interaction was due to the increase of the dual-task costs with RTs, from the 1st to the 5th quintile: $-16,-24,-30,-46$, and $-69 \mathrm{~ms}, t \mathrm{~s}(20)>3.30, p \mathrm{~s}<.01$. All other effects were not significant, $F_{\mathrm{S}}<2.00, p \mathrm{~s}>.10$.

\section{Discussion}

With a CTOA of $300 \mathrm{~ms}$, we observed IOR in manual RTs and dual-task costs in movement times. The effect of IOR and the dual-task costs increased with increasing RTs. The results were thus very similar to those obtained with saccadic responses in Experiment 1. In particular, again we found IOR to color-singleton cues among the slower responses and failed to observe significant interactions between cue relevance and validity as these were predicted by contingent capture theory (predicting capture and/or IOR only in relevant conditions; $\mathrm{cf}$. Gibson \& Amelio, 2000).

Therefore, whether one uses saccadic or manual responses is in itself not responsible for IOR. The same conclusion has been reached in past research (e.g., Souto \& Kerzel, 2009), although some aspects of saccadic IOR are of a different origin than manual IOR effects and might not be due to attention (cf. Hunt \& Kingstone, 2003; Tian, Klein, Satel, Xu, \& Yao, 2011).

In the present experiment, this conclusion is tempered, however, by the fact that task requirements were similar for saccadic and manual responses. In both cases, the task required target localization, and not target discrimination (cf. Gabay, Chica,

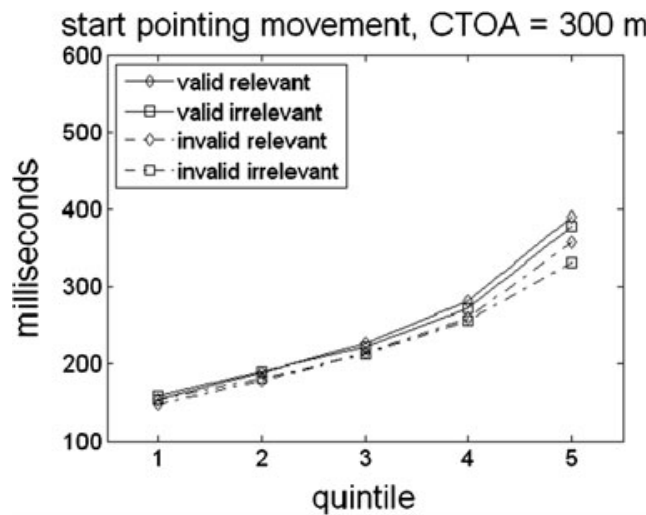

Fig. 4 Experiment 3: On the left are the starting times of the pointing movements (in milliseconds) as a function of the cue's relevance, validity, and the quintile of the starting time distribution. On the right, we plotted

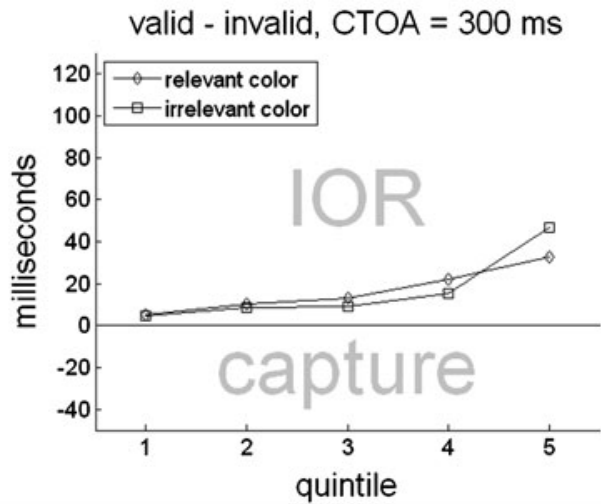

the starting times in the valid minus invalid conditions. Inhibition of return (IOR) results in positive values, and attentional capture in negative values 
Charras, Funes, \& Henik, 2012). Hence, one might argue that our manual responses and saccadic responses were equally sensitive to nonattentional motor effects (i.e., priming of a goal-directed response by the cue) and insensitive to attentional capture and IOR effects. Also, a second critical difference to past research concerned the use of a dual task (discrimination and saccade execution) instead of a single task (e.g., discrimination in Gibson \& Amelio, 2000).

\section{Experiment 4}

To understand the influence of task and type of required response, we ran a single-task version of the discrimination task with manual button-press responses (Gibson \& Amelio, 2000). In addition, because past research used CTOA as a withinparticipant variable (Gibson \& Amelio, 2000), this was also done in Experiment 4 in which the CTOA was $100 \mathrm{~ms}, 200 \mathrm{~ms}$, or $1 \mathrm{~s}$.

\section{Method}

Participants Twelve (six male, six female), students, with a mean age of 23 years, participated in Experiment 4. They had normal or corrected-to-normal visual acuity. They received course credit in exchange for their participation.

Apparatus, stimuli, and procedure These were similar to Experiments 1 to 3 with the following exceptions. Balanced across participants, the target could either be red, green, or blue. A matching or nonmatching cue was presented with a CTOA of $100 \mathrm{~ms}, 200 \mathrm{~ms}$, or $1 \mathrm{~s}$. The matching cue had the same color as the searched-for target (e.g., it was red if the target was red) and the nonmatching cue had a different color than the target (e.g., it was blue if the target was red), with cue colors balanced across participants. The cues were not associated with any task and could be ignored. Cue position did not predict the target position. Participants searched for the color-defined target and reported its shape (whether it was an E or a digital 3 by pressing one of two buttons).

\section{Results}

Manual RTs For the results, see also Fig. 5. Correct RTs were submitted to a three-way ANOVA with the within-participants variables cue type (matching cue, nonmatching cue), validity (valid, invalid), quintile (or bin), and CTOA (100 ms, $200 \mathrm{ms,}$ $1 \mathrm{~s})$. The ANOVA revealed a significant main effect of validity, $F(1,11)=29.06, p<.01$, reflecting faster valid than invalid RTs (558 vs. $577 \mathrm{~ms})$. That is, there was attentional capture of $-19 \mathrm{~ms}$. Significant interactions of validity and cue type, $F(1,11)=17.10, p<.01$, and of validity and CTOA, $F(2,22)=3.51, p<.05$, were due to stronger capture effects with the matching cues $(-45 \mathrm{~ms}), t(11)=5.55, p<.01$, than with the nonmatching cues $(-4 \mathrm{~ms}), t(11)=1.03, p=.33$, and to stronger capture effects with short CTOAs [at $100 \mathrm{~ms}$, $-37 \mathrm{~ms}, t(11)=4.03, p<.01$; at $200 \mathrm{~ms},-20 \mathrm{~ms}, t(11)=$ $6.17, p<.01$ ] than with the long CTOA [at $1,000 \mathrm{~ms},-12 \mathrm{~ms}$; $t(11)=2.71, p<.05]$. Finally, we found a significant three-way interaction of validity, relevance, and CTOA, $F(2,22)=8.72$, $p<.01$, that was even further qualified by a significant fourway interaction of all variables, $F(4,44)=2.47, p<.05$. The four-way interaction showed that in the matching conditions, significant capture effects were found with all CTOAs and all quintiles of the RT distribution [ts $(11)>2.70, p \mathrm{~s}<.05$ ], with the exception of the slowest responses (3rd to 5 th quintile) in the longest CTOA of $1 \mathrm{~s}[t \mathrm{~s}(11)<1.90, p \mathrm{~s}>.08]$, whereas in the nonmatching conditions, no capture was found in all CTOAs and all quintiles [ts $(11)<1.70, p \mathrm{~s}>.11]$, with the exception of the fastest responses (1st quintile) of the shortest CTOA of $100 \mathrm{~ms}[-12 \mathrm{~ms} ; t(11)=3.45, p<.01]$.

Errors Because the numbers or errors were sufficient for formal analyses, we conducted ANOVAs of the error rates with the variables, cue type (matching cue, nonmatching cue), validity (valid, invalid), and CTOA (100, 200, 1,000 ms). For this analysis, we summed the participants' failures to respond and their erroneous responses. Missing erroneous trials were repeated at a random point later in the experiment. Participants made $8.9 \%$ errors in total. An ANOVA of these error rates did not reveal any significant effects.

\section{Discussion}

In line with Gibson and Amelio (2000), there was no IOR in a buttonpress discrimination task. Also in line with Gibson and Amelio, there was attentional capture by matching cues, but not by nonmatching cues. Yet, in agreement with the possibility of deallocation of attention, capture by matching cues decreased with longer CTOAs and became nonsignificant among the slowest responses of the longest CTOA of $1 \mathrm{~s}$. However, in contrast to Gibson and Amelio's findings, we also found a smaller but significant capture effect by the irrelevant cues among the fastest responses of the shortest CTOA. Because we used a quintile analysis that was not used by Gibson and Amelio, it is possible that they overlooked this on average tiny effect (see also van Zoest et al., 2004).

\section{Experiment 5}

To see whether the use of saccades accounted for the presence of IOR, we also ran a single-task version of the saccade task. Otherwise procedures were as in the manual task of Experiment 4. 

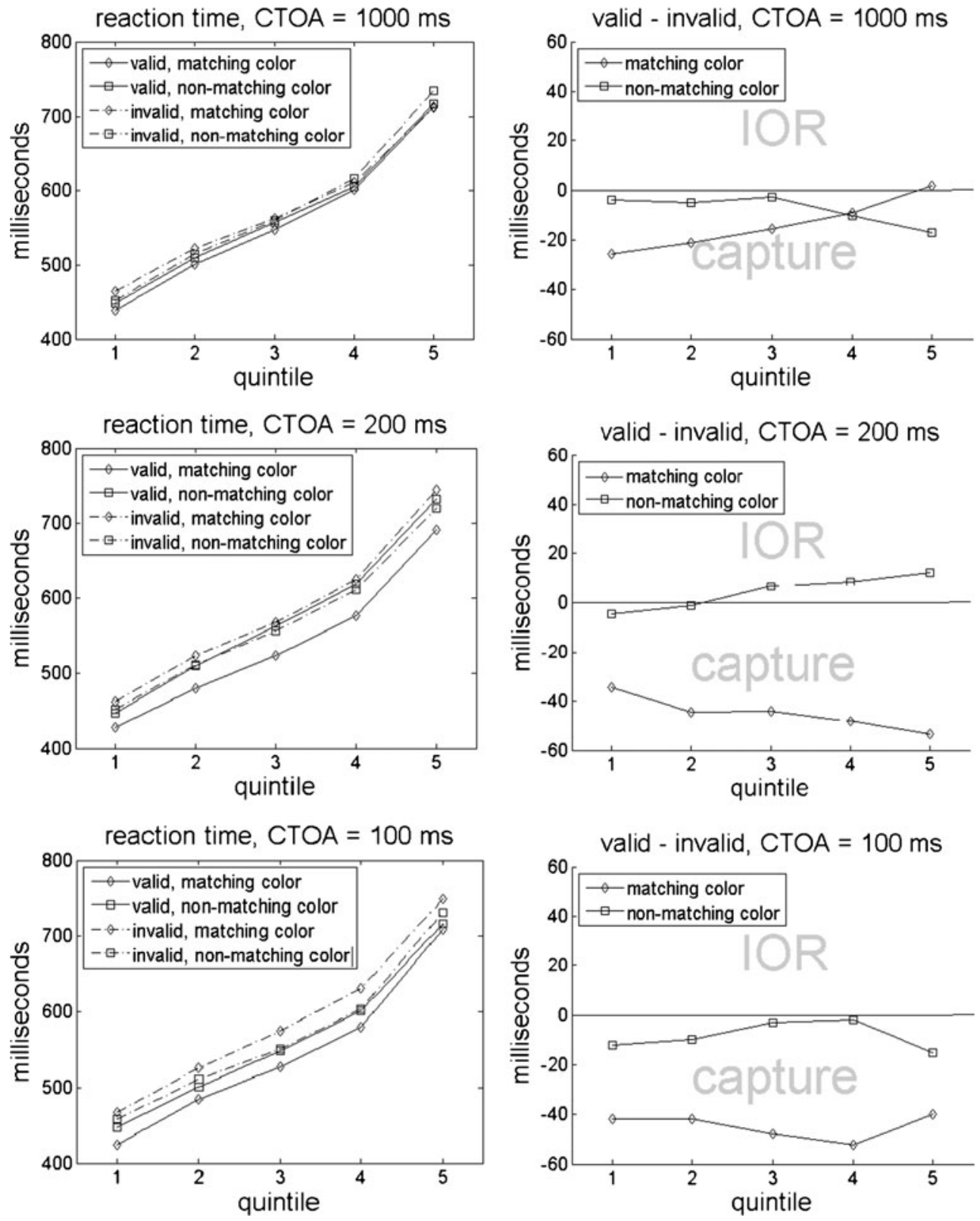

Fig. 5 On the left are manual reaction times (RTs; in milliseconds) in Experiment 4, as a function of the cue's matching to the top-down set, its validity, and the quintile of the RT distribution. The RTs were measured under conditions with cue-target onset asynchronies of $1 \mathrm{~s}$ (upper panels), $200 \mathrm{~ms}$ (middle panels), and of $100 \mathrm{~ms}$ (lower panels). On the right, we

\section{Method}

Participants Twelve (six male, six female) students, with a mean age of 23 years participated in Experiment 5. They had normal or corrected-to-normal visual acuity. They received course credit in exchange for their participation.

Apparatus, stimuli, and procedure These were similar to Experiment 4, with the exception that participants searched

have plotted inhibition of return (IOR, depicted above the $x$-axis) and capture (below the $x$-axis) (calculated as RT in the valid minus RT in the invalid conditions) as a function of the cue's matching to the top-down set and the quintile of the RT distribution

for a predefined color target and made a saccade to it as quickly and accurately as possible.

\section{Results}

Participants made $8.3 \%$ errors in total. Of all responses, $3.5 \%$ were discarded because they were faster than $100 \mathrm{~ms}$, or because of equipment malfunctioning. 
Saccadic RTs For the results, see also Fig. 6. The same threeway ANOVA as in Experiment 4 on mean correct saccadic RTs led to a significant main effect of CTOA, $F(2,11)=$ $35.90, p<.01$, with longer latencies at short CTOAs (at $100 \mathrm{~ms}, 323 \mathrm{~ms}$; at $200 \mathrm{~ms}, 327 \mathrm{~ms}$ ) than at long CTOAs (at $1,000 \mathrm{~ms}, 279 \mathrm{~ms}$ ), which is probably related to response preparation. Also, this analysis revealed significant two-way interactions of validity and cue type, $F(1,11)=9.11, p<.01$, with more capture by matching $[-13 \mathrm{~ms}, t(11)=2.49, p<.05]$ than by nonmatching $[-2 \mathrm{~ms}, t<1.00]$ cues, an interaction of
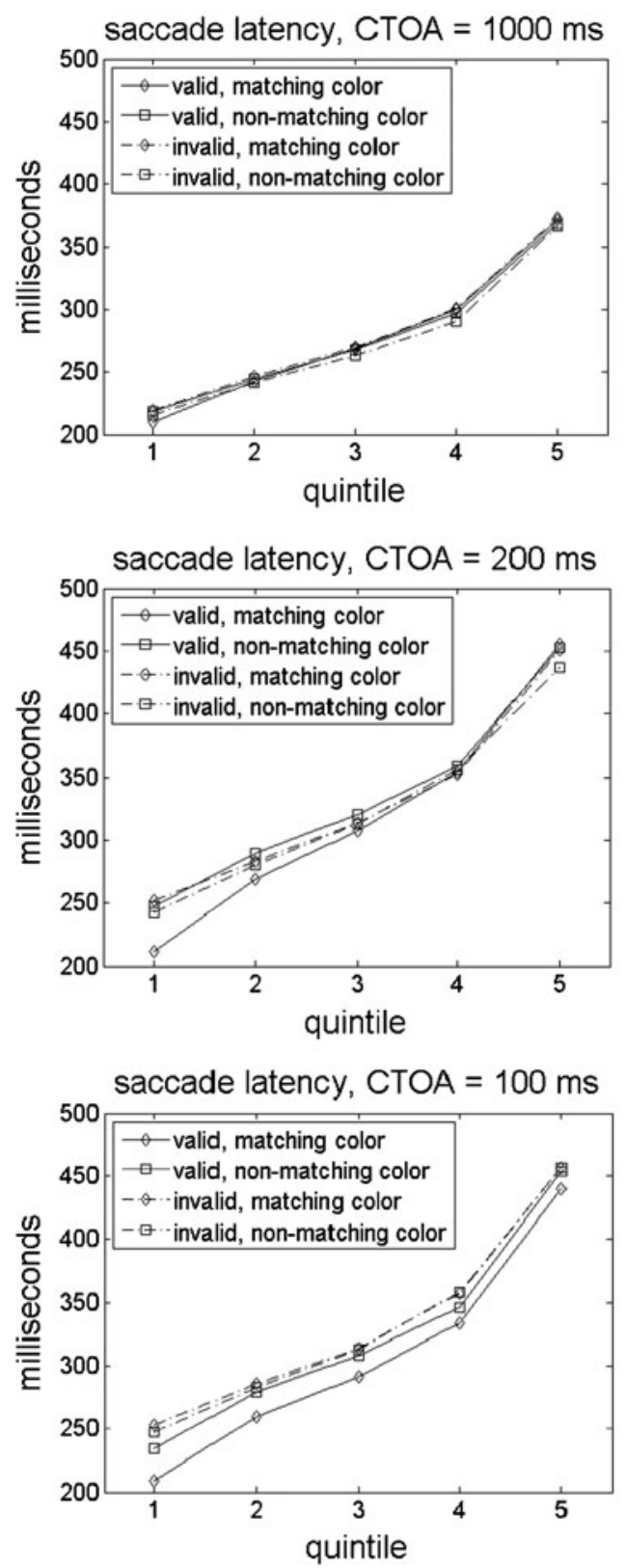

Fig. 6 On the left are saccadic latencies (in milliseconds) in Experiment 5, as a function of the cue's matching to the top-down set, its validity, and the quintile of the RT distribution. The saccadic RTs were measured under conditions with cue-target onset asynchronies of $1 \mathrm{~s}$ (upper panels), $200 \mathrm{~ms}$ (middle panels), and $100 \mathrm{~ms}$ (lower panels). On the right, we have plotted validity and CTOA, $F(2,11)=7.92, p<.01$, with more capture in the shortest CTOA [at $100 \mathrm{~ms},-18 \mathrm{~ms}, t(11)=$ $3.52, p<.01]$ than in the two longer CTOAs (at $200 \mathrm{~ms}$, $-2 \mathrm{~ms}$; at $1,000 \mathrm{~ms}, 0 \mathrm{~ms}$; both $t \mathrm{~s}<1.00$ ), and an interaction of validity and quintile, $F(4,11)=5.46, p<.05$, with evidence for capture restricted to the fastest responses [1st quintile: $-17 \mathrm{~ms}$, $t(11)=3.90, p<.01]$, and absent for all other quintiles [2nd to 5th quintile: $-6,-4,-4$, and $0 \mathrm{~ms}, t \mathrm{~s}(11)<1.40, p \mathrm{~s}>.19$ ]. Finally, a significant three-way interaction of validity, relevance, and CTOA, $F(4,44)=3.98, p<.01$, and a significant
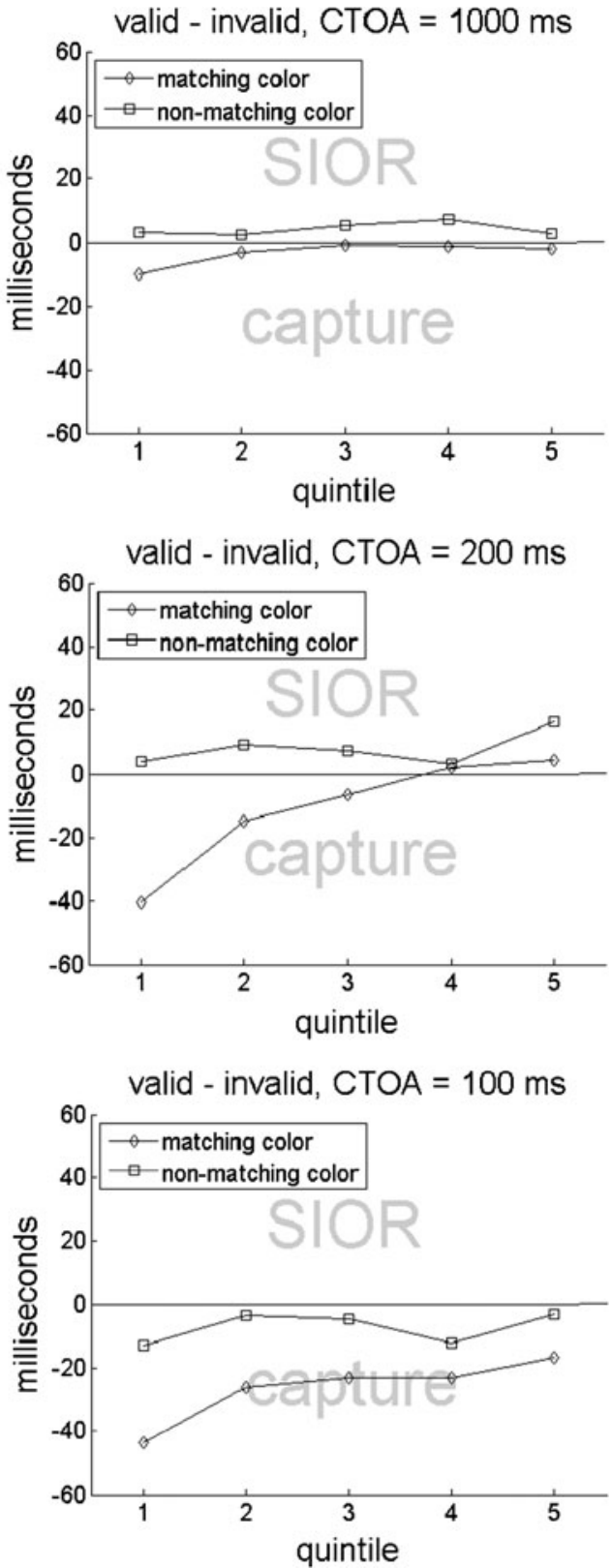

inhibition of return (IOR, depicted above the $x$-axis) and capture (below the $x$-axis) (calculated as RT in the different- minus RT in the same-position conditions) as a function of the cue's matching to the top-down set and the quintile of the RT distribution 
four-way interaction of all variables, $F(8,88)=2.47, p<.05$, were found. They showed that only the matching cues and fast responses led to a capture effect [1st quintile, $-31 \mathrm{~ms}, t(11)=$ $4.98, p<.01 ; 2$ nd quintile, $-14 \mathrm{~ms}, t(11)=2.43, p<.05]$, whereas all other quintiles of the matching conditions [3rd, 4 th, and 5th: $-10,-8$, and $-7 \mathrm{~ms}$, respectively; ts $(11)<1.70, p \mathrm{~s}>.12]$, and the nonmatching conditions were not significant [1st to 5th: $-2,2,3,1$, and $5 \mathrm{~ms}$, respectively; $t \mathrm{~s}(11)<1.20, p \mathrm{~s}>.27]$.

Errors Next, erroneous saccades in the invalid condition were subjected to an ANOVA with the variables cue type (matching vs. non-matching), saccade direction (to the cued position vs. away from the cued position) ${ }^{1}$, and CTOA (100 ms, $200 \mathrm{~ms}$, $1 \mathrm{~s})$. There was a significant main effect of cue type, $F(1,11)=$ $5.19, p<.05$, reflecting higher error rates to matching than to nonmatching cues $(4.62 \%$ vs. $3.36 \%)$. There were also significant interactions of direction and CTOA, $F(2,22)=$ $6.11, p<.01$, and of relevance, direction, and CTOA, $F(2$, $22)=4.63, p<.05$. The two-way interaction reflected differences between error rates at cued locations minus error rates at uncued locations of $-2.45 \%$ and of $-3.37 \%$ in the $200-\mathrm{ms}$ and $1 \mathrm{~s}$ CTOAs, respectively, $t \mathrm{~s}>|2.40|, p \mathrm{~s}<.05$. There was no significant difference between the prevalence of erroneous saccades toward the cue and away from the cue $(2.25 \% \mathrm{vs}$. $2.17 \%$ ) in the 100-ms CTOA, $t<1.00$.

The three-way interaction reflected a tendency to move toward the uncued position in the nonmatching conditions that increased with CTOA [at $100 \mathrm{~ms},-1.53 \%$, n.s.; at $200 \mathrm{~ms},-2.65 \% ; t(11)=-2.51, p<.05]$. In contrast, we observed a tendency to move to the cued location in the matching condition that decreased with CTOA [at $100 \mathrm{~ms}$, $1.65 \%$, n.s.; at $200 \mathrm{~ms},-2.27 \%$; $t(11)=2.20, p=.05]$. With a CTOA of $1 \mathrm{~s}$, errors away from the cued location were equally frequent for matching cues and nonmatching cues $(-3.58 \%$ vs. $-3.14 \%), t \mathrm{~s}(11)>|2.30|, p \mathrm{~s}<.05$.

\section{Discussion}

Again, we found evidence for stronger capture effects in the matching than in the nonmatching conditions. In line with the contingent capture view, among the fastest responses, capture effects were restricted to the matching cueing conditions (see Ansorge \& Horstmann, 2007; Burnham, 2013). Again, however, it is equally possible that intertrial priming increased the capture effect in the matching conditions. Further, capture was stronger with short CTOAs.

\section{General discussion}

In the present study, we used saccadic responses (in Exps. 1, 2, and 5) and manual responses (in Exps. 3 and 4) to test (1) whether color singletons lead to saccadic and manual IOR, and (2) whether saccadic IOR was different with relevant (or matching) and irrelevant (or nonmatching) color singletons.

In Experiments 1-3, half of the cues were relevant because observers had to memorize the cue position and perform a discrimination task at the cued location. In Experiments 1 and 3 , neither cue color matched the target color, whereas the relevant cue color did match the target color in Experiment 2. These experiments yielded two main results: First, cueing effects were similar with relevant and irrelevant cues, even though RTs were longer with relevant cues. Second, RTs were mostly longer at the cued location, indicating IOR, and the analysis of RT quintiles shows that this effect increased for slower responses. For the range of CTOAs tested (200-500 ms), no modulation of IOR by CTOA was apparent. Although we mostly observed IOR in Experiments 1-3, attentional capture occurred with rapid responses at a short CTOA (Exp. 1), in particular when the relevant cue matched the target color (Exp. 2). Overall, however, both relevant and irrelevant cues mostly produced IOR when the cue color did not match the target color. IOR was strongest for slow responses and long CTOAs.

In Experiments 4-5, all cues were irrelevant and half of the cues matched the target color whereas the other half did not. The results were markedly different from the first set of experiments. Most importantly, IOR disappeared and was not even significant for the slowest responses and the longest CTOAs. Also, we found a marked difference between matching and nonmatching cues: Whereas neither IOR nor attentional capture occurred for nonmatching cues, we did find strong attentional capture for matching cues. Attentional capture for matching cues was particularly strong for short CTOAs (both manual and saccadic responses) and rapid responses (only saccadic responses). In contrast to Gibson and Amelio (2000), we did observe attentional capture with a CTOA of $1 \mathrm{~s}$ and manual responses, at least for fast RTs (Exp. 4). However, attentional capture was absent altogether for saccadic responses and a CTOA of $1 \mathrm{~s}$ (Exp. 5).

Therefore, the response as to whether saccadic and manual IOR to color singletons exists is modulated by the experimental protocol, CTOA, and RT quintile. This may explain the inconsistencies in the literature. IOR is observed when the cues are partly task-relevant (though not predictive of the target location) even at relatively short CTOAs of 200 $500 \mathrm{~ms}$, whereas capture is observed with completely taskirrelevant cues that match the color of the target up to CTOAs of $1 \mathrm{~s}$. Furthermore, IOR is greatest in slow responses, whereas capture is greatest with fast responses, which is consistent with the original idea of a sequence of attentional orienting followed by inhibition.

The complexity of the data pattern may explain why previous research has yielded inconsistent results. Some authors found saccadic IOR with color singletons (cf. Godijn \& Theeuwes, 2004), but a majority of studies failed to find 
IOR in manual responses with color singletons (cf. Gibson \& Amelio, 2000; Remington et al., 2001). In the present study, we were able to demonstrate saccadic IOR in saccadic RTs (Exps. 1 and 2) and in manual pointing RTs (Exp. 3) to color singletons in a task in which half of the cues were relevant. However, in line with prior findings by Gibson and Amelio, IOR in manual and saccadic RTs could not be observed when the cues were completely task-irrelevant (Exps. 4 and 5).

The present study also provided a clue as to why IOR of color singletons might have been overlooked in past studies: The IOR was most prominent among the slower responses. In Experiment 2, we even found capture effects (i.e., advantages for valid relative to invalid cues) in the faster responses, and saccadic IOR was restricted to the slower responses (see Fig. 3). Thus, saccadic and manual IOR can cancel one another out and might be overlooked if one averages across fast and slow responses. (For an example, consider the CTOA of 300 ms of Exp. 1 in Fig. 2.).

With respect to the second question that we investigated, some researchers argued that more deallocation could be found with irrelevant cues than with relevant cues (Theeuwes, 2010). According to the deallocation explanation, the different speed with which attention can be withdrawn from irrelevant versus relevant cues leads to more measurable cueing with relevant than irrelevant cues (cf. Theeuwes, 2010; Theeuwes et al., 2000). This prediction could not be confirmed in all past studies. Gibson and Amelio (2000), for example, were unable to find any indices of deallocation after irrelevant color cues. This was in marked contrast to their observations with relevant onset cues, in which these authors found deallocation in the form of IOR.

In the present research, we therefore set out to test this important prediction of the deallocation explanation. Here, we used saccadic IOR as a hallmark of deallocation. In line with the deallocation explanation, the interaction of relevance, validity and quintile in Experiment 2 suggests that the increase of saccadic IOR or conversely, the offset of capture, across the RT distribution is particularly rapid when the cue color is unequal to the target color.

Our study also provided a number of clues as to why past research might have overlooked deallocation. First of all, the number of relevant colors proved to be influential. When more than one relevant color was used, the indications of top-down contingent capture and of differential saccadic IOR were numerically weak. This was found in a comparison of Experiment 1, in which we used two relevant colors (one for the relevant cue and one for the target), with Experiment 2 in which we used one relevant color. The different strengths of capture and subsequent saccadic IOR in Experiments 1 and 2 resonate with the known reluctance of the participants to search for colors if singleton search offers an easier way to find the targets (cf. Folk \& Anderson, 2010).
Another important variable for whether one finds differential deallocation was the CTOA. In Experiment 1, with a CTOA of $300 \mathrm{~ms}$ (a CTOA that is not uncommon in IOR research because at least manual IOR would only commence at about this CTOA; cf. Klein, 2000), and even less so with a CTOA of 500 ms (in Exp. 1), we were not able to find any traces of differential deallocation in saccadic RTs. Only when the CTOA was reduced to $200 \mathrm{~ms}$ (e.g., in Exp. 2) were we able to find more deallocation after irrelevant than relevant color-singleton cues. This conclusion was also backed up in Experiment 5 in which differential deallocation was restricted to the small CTOAs. Because CTOAs were longer in some past studies (cf. Gibson \& Amelio, 2000) our findings also provided an insight into why the differential IOR effect could have been missed in these studies.

Finally, again, saccadic latency, or more precisely, the quintile of the saccadic RT distribution, proved critical because more saccadic IOR to irrelevant/nonmatching than to relevant/matching cues was most clearly seen among the fastest saccades (of Exp. 2). Among the slower saccades, by contrast, differential saccadic IOR was washed out and no significant interaction of the variables cue relevance and cue position was found anymore (Exp. 2).

In addition, as might be expected, with the exception of the CTOA of $500 \mathrm{~ms}$, the more difficult dual-task condition, with the relevant cues and the discrimination task at the end of the trial, interfered with response execution in Experiments 1 to 3 (cf. Carbone \& Schneider, 2010). That is, RTs were longer with relevant cues that implied a secondary memory task than with irrelevant cues. Note that our relevance manipulation affected response latencies mostly during the slower responses. This also offers a clue as to why the same interference effect by the secondary (cue memorization) task on saccadic RT was not found with a CTOA of $500 \mathrm{~ms}$ (in Exp. 1). A CTOA of $500 \mathrm{~ms}$ was probably too long to still show the detrimental overall effect of the more demanding task of first having to attend to the cue and then having to make a saccade to the target because at the time that a capacity-limited mechanism was needed for an attention shift toward the saccade target this mechanism was no longer engaged by the shifting of attention to the relevant cue and for encoding it into working memory (cf. Pashler, Carrier, \& Hoffman, 1993).

In conclusion, the present study demonstrated the presence of saccadic IOR to color singletons and showed that button presses are insensitive to this sort of IOR. Furthermore, the saccadic IOR effects with a single relevant color provided one of the rare pieces of evidence for Theeuwes et al.'s (2000) deallocation explanation (but of course, this would not mean that other top-down factors - such as contingent capture - can be excluded).

Author Note First authorship was shared by U.A. and H.-W.P., Faculty of Psychology, University of Vienna, Vienna, Austria. The research 
was supported by Project No. CS11-009 of the WWTF (Wiener Wissenschafts- und Technologiefonds) to U.A., Otmar Scherzer, and Shelley Buchinger.

\section{References}

Abrams, R. A., \& Dobkin, R. S. (1994). Inhibition of return: Effects of attentional cuing on eye movement latencies. Journal of Experimental Psychology. Human Perception and Performance, 20, 467-477. doi:10.1037/0096-1523.20.3.467

Anderson, B. A., \& Folk, C. L. (2010). Variations in the magnitude of attentional capture: Testing a two-process model. Attention, Perception, \& Psychophysics, 72, 342-352. doi:10.3758/APP.72.2.342

Ansorge, U., \& Horstmann, G. (2007). Preemptive control of attentional capture by color: Evidence from trial-by-trial analysis and ordering of onsets of capture effects in RT distributions. Quarterly Journal of Experimental Psychology, 60, 952-975.

Ansorge, U., Kiss, M., Worschech, F., \& Eimer, M. (2011). The initial stage of visual selection is controlled by top-down task set: New ERP evidence. Attention, Perception, \& Psychophysics, 73, 113-122.

Awh, E., Vogel, E. K., \& Oh, S. H. (2006). Interactions between attention and working memory. Neuroscience, 139, 201-208. doi:10.1016/j. neuroscience.2005.08.023

Belopolsky, A. V., \& Theeuwes, J. (2009). Inhibition of saccadic eye movements to locations in spatial working memory. Attention, Perception, \& Psychophysics, 71, 620-631. doi:10.3758/APP.71.3.620

Belopolsky, A. V., \& Theeuwes, J. (2011). Selection within visual memory representations activates the oculomotor system. Neuropsychologia, 49, $1605-1610$

Briand, K. A., Larrison, A. L., \& Sereno, A. B. (2000). Inhibition of return in manual and saccadic response systems. Perception \& Psychophysics, 62, 1512-1524.

Burnham, B. R. (2007). Displaywide visual features associated with a search display's appearance can mediate attentional capture. Psychonomic Bulletin \& Review, 14, 392-422. doi:10.3758/BF03194082

Burnham, B. R. (2013). Using response-time distributions to examine top-down influences of attentional capture. Attention, Perception, \& Psychophysics, 75, 257-277.

Carbone, E., \& Schneider, W. X. (2010). The control of stimulus-driven saccades is subject not to central, but to visual attention limitations. Attention, Perception, \& Psychophysics, 72, 2168-2175.

Cole, G. G., Kuhn, G., Heywood, C. A., \& Kentridge, R. W. (2009). The prioritization of feature singletons in the allocation of visual attention. Experimental Psychology, 56, 134-146.

Deubel, H. (2008). The time course of presaccadic attention shifts. Psychological Research, 72, 630-640.

Deubel, H., \& Schneider, W. X. (1996). Saccade target selection and object recognition: Evidence for a common attentional mechanism. Vision Research, 36, 1827-1837. doi:10.1016/0042-6989(95)00294-4

Dorris, M. C., Klein, R. M., Everling, S., \& Munoz, D. P. (2002). Contribution of the primate superior colliculus to inhibition of return. Journal of Cognitive Neuroscience, 14, 1256-1263. doi:10. $1162 / 089892902760807249$

Eimer, M., \& Kiss, M. (2008). Involuntary attentional capture is determined by task set: Evidence from event-related brain potentials. Journal of Cognitive Neuroscience, 20, 1423-1433. doi:10.1162/ jocn.2008.20099

Folk, C. L., \& Anderson, B. A. (2010). Target-uncertainty effects in attentional capture: Color-singleton set or multiple attentional control settings? Psychonomic Bulletin \& Review, 17, 421-426. doi:10. 3758/PBR.17.3.421

Folk, C. L., \& Remington, R. (1998). Selectivity in distraction by irrelevant featural singletons: Evidence for two forms of attentional capture. Journal of Experimental Psychology. Human Perception and Performance, 24, 847-858. doi:10.1037/0096-1523.24.3.847

Folk, C. L., Remington, R. W., \& Johnston, J. C. (1992). Involuntary covert orienting is contingent on attentional control settings. Journal of Experimental Psychology. Human Perception and Performance, 18, 1030-1044. doi:10.1037/0096-1523.18.4.1030

Gabay, S., Chica, A. B., Charras, P., Funes, M. J., \& Henik, A. (2012). Cue and target processing modulate the onset of inhibition of return. Journal of Experimental Psychology. Human Perception and Performance, 38, 42-52.

Gibson, B. S., \& Amelio, J. (2000). Inhibition of return and attentional control settings. Perception \& Psychophysics, 62, 496-504.

Godijn, R., \& Theeuwes, J. (2004). The relationship between inhibition of return and saccade trajectory deviations. Journal of Experimental Psychology. Human Perception and Performance, 30, 538-554.

Grubert, A., \& Eimer, M, (2013). Qualitative differences in the guidance of attention during single-color and multiple-color visual search: Behavioral and electrophysiological evidence. Journal of Experimental Psychology: Human Perception and Performance. doi:10. 1037/a0031046

Hunt, A. R., \& Kingstone, A. (2003). Inhibition of return: Dissociating attentional and oculomotor components. Journal of Experimental Psychology. Human Perception and Performance, 29, 1068-1074. doi:10.1037/0096-1523.29.5.1068

Irons, J. L., Folk, C. L., \& Remington, R. W. (2012). All set! Evidence of simultaneous attentional control settings for multiple target colors. Journal of Experimental Psychology. Human Perception and Performance, 38, 758-775.

Itti, L., Koch, C., \& Niebur, E. (1998). A model of saliency-based visual attention for rapid scene analysis. IEEE Transactions on Pattern Analysis and Machine Intelligence, 20, 1254-1259.

Kim, M.-S., \& Cave, K. R. (1999). Top-down and bottom-up attentional control: On the nature of interference from a salient distractor. Perception \& Psychophysics, 61, 1009-1023. doi:10.3758/BF03207609

Klein, R. M. (2000). Inhibition of return. Trends in Cognitive Sciences, 4, 138-147. doi:10.1016/S1364-6613(00)01452-2

Lamy, D. (2010). Reevaluating the disengagement hypothesis. Acta Psychologica, 135(2), 127-129.

Lamy, D., Leber, A., \& Egeth, H. E. (2004). Effects of task relevance and stimulus-driven salience in feature-search mode. Journal of Experimental Psychology. Human Perception and Performance, 30, 1019-1031. doi:10.1037/0096-1523.30.6.1019

Lupiàñez, J. (2010). Inhibition of return. In A. C. Nobre \& J. T. Coull (Eds.), Attention and time (pp. 17-34). Oxford, UK: Oxford University Press.

Maljkovic, V., \& Nakayama, K. (1994). Priming of pop-out: I. Role of features. Memory and Cognition, 22, 657-672. doi:10.3758/ BF03209251

Pashler, H., Carrier, M., \& Hoffman, J. (1993). Saccadic eye-movements and dual-task interference. Quarterly Journal of Experimental Psychology, 46A, 51-82.

Posner, M. I., \& Cohen, Y. (1984). Components of visual orienting. In H. Bouma \& D. G. Bouwhuis (Eds.), Attention and performance X: Control of language processes (pp. 531-556). Hillsdale, NJ: Erlbaum.

Pratt, J., \& McAuliffe, J. (2002). Determining whether attentional control settings are inclusive or exclusive. Perception \& Psychophysics, 64 1361-1370. doi:10.3758/BF03194778

Remington, R. W., Folk, C. W., \& McLean, J. P. (2001). Contingent attentional capture or delayed allocation of attention? Perception \& Psychophysics, 63, 298-307.

Schreij, D., Owens, C., \& Theeuwes, J. (2008). Abrupt onsets capture attention independent of top-down control settings. Perception \& Psychophysics, 70, 208-218. doi:10.3758/PP.70.2.208

Schreij, D., Theeuwes, J., \& Olivers, C. N. L. (2010a). Abrupt onsets capture attention independent of top-down control settings II: Additivity is no evidence for filtering. Attention, Perception, \& Psychophysics, 72, 672-682. doi:10.3758/APP.72.3.672 
Schreij, D., Theeuwes, J., \& Olivers, C. N. L. (2010b). Irrelevant onsets cause inhibition of return regardless of attentional set. Attention, Perception, \& Psychophysics, 72, 1725-1729. doi:10.3758/APP. 72.7.1725

Sokal, R. R., \& Rohlf, J. F. (1981). Biometry: The principles and practice of statistics in biological research (2nd ed.). San Francisco, CA: W. H. Freeman.

Souto, D., \& Kerzel, D. (2009). Evidence for an attentional component in saccadic inhibition of return. Experimental Brain Research, 195, $531-540$

Taylor, T. L., \& Klein, R. M. (1998). On the cause and effects of inhibition of return. Psychonomic Bulletin and Review, 5, 625-643.

Theeuwes, J. (1992). Perceptual selectivity for color and form. Perception \& Psychophysics, 51, 599-606. doi:10.3758/BF03211656

Theeuwes, J. (2010). Top-down and bottom-up control of visual selection. Acta Psychologica, 123, 77-99. doi:10.1016/j.actpsy.2010.02.006

Theeuwes, J., Atchley, P., \& Kramer, A. F. (2000). On the time course of top-down and bottom-up control of visual attention. In S. Monsell \& J. Driver (Eds.), Control of cognitive processes:
Attention and performance XVIII (pp. 105-125). Cambridge, MA: MIT Press.

Tian, Y., Klein, R. M., Satel, J., Xu, P., \& Yao, D. (2011). Electrophysiological explorations of the cause and effect of inhibition of return in a cue-target paradigm: A spatio-temporal theory. Brain Topography, 24, 164-182.

van Zoest, W., Donk, M., \& Theeuwes, J. (2004). The role of stimulusdriven and goal-driven control in saccadic visual selection. Journal of Experimental Psychology. Human Perception and Performance, 30, 746-759. doi:10.1037/0096-1523.30.4.749

Wang, Z., \& Klein, R. M. (2010). Searching for inhibition of return in visual search-A review. Vision Research, 50, 220-228. doi:10. 1016/j.visres.2009.11.013

Worschech, F., \& Ansorge, U. (2012). Top-down search for color prevents voluntary directing of attention to informative singleton cues. Experimental Psychology, 59, 153-162.

Zhang, Y., \& Zhang, M. (2011). Spatial working memory load impairs manual but not saccadic inhibition of return. Vision Research, 51, 147-153. doi:10.1016/j.visres.2010.10.022 